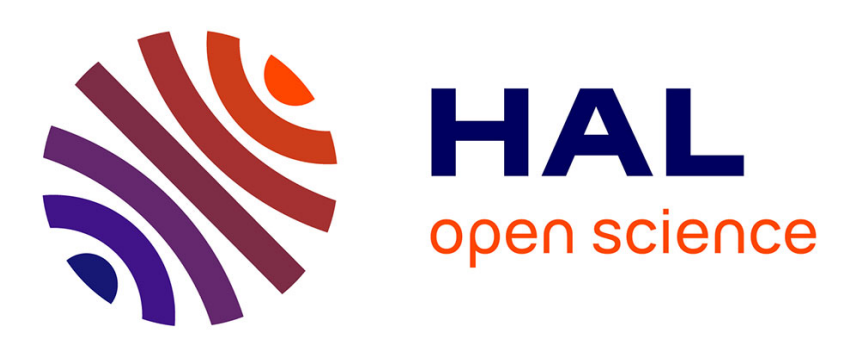

\title{
Upscaled model for diffusion and serial reduction pathways in porous electrodes
}

T.D. Le, L. Zhang, A. Kuhn, N. Mano, G. Vignoles, Didier Lasseux

\section{To cite this version:}

T.D. Le, L. Zhang, A. Kuhn, N. Mano, G. Vignoles, et al.. Upscaled model for diffusion and serial reduction pathways in porous electrodes. Journal of Electroanalytical Chemistry, 2019, pp.113325. 10.1016/j.jelechem.2019.113325 . hal-02368380

\section{HAL Id: hal-02368380 \\ https://hal.science/hal-02368380}

Submitted on 18 Nov 2019

HAL is a multi-disciplinary open access archive for the deposit and dissemination of scientific research documents, whether they are published or not. The documents may come from teaching and research institutions in France or abroad, or from public or private research centers.
L'archive ouverte pluridisciplinaire HAL, est destinée au dépôt et à la diffusion de documents scientifiques de niveau recherche, publiés ou non, émanant des établissements d'enseignement et de recherche français ou étrangers, des laboratoires publics ou privés. 


\title{
Upscaled model for diffusion and serial reduction pathways in porous electrodes
}

\author{
T. D. Le \\ Institut de Mécanique et d'Ingénierie, CNRS UMR5295, Esplanade des Arts et Métiers, \\ Talence Cedex 33405, France \\ L. Zhang \\ Institut des Sciences Moléculaires, CNRS UMR5255, University of Bordeaux ENSCBP, \\ 16 Avenue Pey Berland, 33607 Pessac, France \\ A. Kuhn \\ Institut des Sciences Moléculaires, CNRS UMR5255, University of Bordeaux ENSCBP, \\ 16 Avenue Pey Berland, 33607 Pessac, France \\ N. Mano \\ Centre de Recherche Paul Pascal, CNRS UPR8641, University of Bordeaux, Avenue \\ Albert Schweitzer, 33600 Pessac, France \\ G. Vignoles \\ Laboratoire des Composites ThermoStructuraux, CNRS UMR5801, 3 Allée de la Boétie, \\ 33600 Pessac, France \\ D. Lasseux* \\ Institut de Mécanique et d'Ingénierie, CNRS UMR5295, Esplanade des Arts et Métiers, \\ Talence Cedex 33405, France
}

* Corresponding author

Email addresses: letiendung.esgc@gmail.com (T. D. Le), lin.zhang@outlook.fr (L. Zhang), kuhn@enscbp.fr (A. Kuhn), mano@crpp-bordeaux.cnrs.fr (N. Mano), vinhola@lcts.u-bordeaux.fr (G. Vignoles), didier.lasseux@u-bordeaux.fr (D. Lasseux) 


\begin{abstract}
Multiscale modelling of coupled diffusion and serial reduction reactions in porous micro-electrodes is developed in this work. The governing coupled equations at the pore scale in the case of two reduction reactions, as for instance, the serial reaction pathway for oxygen reduction to hydrogen peroxide and subsequently to water, are upscaled to obtain a macroscopic model describing the process in an effective medium at the electrode scale. This new macroscopic model, obtained from the volume averaging technique, is validated through comparisons with results of 3D Direct Numerical Simulations of the pore-scale model. The excellent agreement between the two approaches proves the relevance of the macroscale model which reduces to a $1 \mathrm{D}$ problem in the configuration under concern, providing a drastic speedup in the computation of the solution. Numerical results obtained with the macroscopic model are successfully compared to experimental data obtained by voltammetry with porous gold electrodes of different thicknesses operating the serial pathway of oxygen reduction to water. Results highlight the ability of this new macroscopic model to predict the electrode behavior and show that the second reduction reaction of hydrogen peroxide plays an important role in the current production.
\end{abstract}

Keywords: Porous micro-electrode, Oxygen reduction reaction, Diffusion reaction macroscopic model, Upscaling, Volume averaging method

\title{
1. Introduction
}

The use of porous electrodes for in vivo implantable active or passive electro-devices is a very promising way for an efficient in situ production of electric energy [1, 2, 3. The main advantage of using a porous material lies in its very large specifc area (pore surface to volume ratio) which favours the heterogeneous electro-chemical reactions of interest [4, 5, 6]. As a consequence, the macroscopic size of the electrode can be significantly reduced and the current can be enhanced by an order of magnitude or more in comparison to a flat electrode of the same size [7, 8].

Important efforts have been dedicated to a better understanding and characterization of such devices operating in different regimes, with the purpose of improving their overall efficiency. Nevertheless, modelling at the macrosopic scale can be quite difficult in particular when catalytic enzymes are embedded in the porous structure [1] for the Direct Electron Transfer 
[9, 10] and Mediated Electron Transfer [11, 12] modes. An optimal design of the architecture of the electrode may be achieved by an analysis of the correlation of the macroscopic current delivered by the electrode with the underlying microscopic structure, electrochemical and mass transfer parameters. Although this might be achieved from Direct Numerical Simulation (DNS) carried out on the pore-scale model, a much more tractable procedure relies on macroscopic modelling [13, 14]. However, even in the simplest case where no enzyme is present, macroscopic modelling has been widely relying on empirical approaches since the early work of Levie [15, later extended by Barcia et al. [16]. For instance, Barnes et al. [17] considered a disk covered by spherical pores operating a single reaction involving one electron and empirically derived a macroscopic model. A similar system (cylindrical pillars regularly distributed and orthogonally positioned on a plane surface), with the same reactional scheme, was recently investigated [18, 19], assuming again an additive contribution of each pore (i.e. independence of the pillars in the diffusion/reaction process). The latter hypothesis was relaxed in the empirical homogenized model proposed by Ender [20] in which the effective diffusivity dependence upon the pore geometry was however not explicitly provided, as in Ferguson and Bazant [21]. An empirical macroscopic model was also used to study a porous rotating disk electrode in the convection- or diffusion-dominated regime [22, 23]. A more formal derivation of a macroscopic model for transport and reaction in a porous electrode, taking into account the solid, fluid and gas phases, was reported by Vidts and White [24], without however any closure allowing an accurate estimate of the effective diffusivity. A new electrode kinetic equation for a non-porous electrode was recently developed on the basis of a coupled model of electron transfer obeying a Butler-Volmer relationship and oxygen mass transfer at steady-state in the case of direct oxygen reduction [25. For a thorough analysis of the macroscopic behavior and, further, in the perspective of an optimization of the devices under concern, a rational approach, based on a cautious derivation of appropriate macroscopic models from the physicoelectrochemical governing equations at the underlying microscopic scale is of major importance. In this context, the volume averaging method was recently employed to obtain a closed macroscopic model operating at the scale of an entire porous electrode, coupling diffusion and reaction [13]. It was further validated with experiments and used to determine the optimal electrode thickness [14. However, this model is limited to the relatively simple case of a single reduction reaction. 
Heterogeneous reduction reactions at a cathodic electrode immersed in an aqueous solution saturated by oxygen constitute a system of common and wide interest [26, 4]. In this configuration, the oxygen reduction shall be considered in different reaction pathways [27]. The first one is the so-called "direct" four-electron reaction

$$
\mathrm{O}_{2}+4 \mathrm{H}^{+}+4 e^{-} \rightarrow 2 \mathrm{H}_{2} \mathrm{O} \quad E_{\mathrm{O}_{2} / \mathrm{H}_{2} \mathrm{O}}^{0}=1.01 \mathrm{~V} \text { vs } \mathrm{Ag} / \mathrm{AgCl}
$$

( $E_{\mathrm{O}_{2} / \mathrm{H}_{2} \mathrm{O}}^{0}$ being the standard potential of the $\mathrm{O}_{2} / \mathrm{H}_{2} \mathrm{O}$ couple). The second one involves two-successive bi-electronic reactions with the intermediate production of hydrogen peroxide. This mechanism is often referred to as a serial (or indirect) reaction pathway. It is such that

$$
\begin{array}{cc}
\mathrm{O}_{2}+2 \mathrm{H}^{+}+2 e^{-} \stackrel{k_{1}}{\rightarrow} \mathrm{H}_{2} \mathrm{O}_{2} & E_{\mathrm{O}_{2} / \mathrm{H}_{2} \mathrm{O}_{2}}^{0}=0.45 \mathrm{~V} \text { vs } \mathrm{Ag} / \mathrm{AgCl} \\
\mathrm{H}_{2} \mathrm{O}_{2}+2 \mathrm{H}^{+}+2 e^{-} \stackrel{k_{2}}{\longrightarrow} 2 \mathrm{H}_{2} \mathrm{O} & E_{\mathrm{H}_{2} \mathrm{O}_{2} / \mathrm{H}_{2} \mathrm{O}}^{0}=1.56 \mathrm{~V} \text { vs } \mathrm{Ag} / \mathrm{AgCl}
\end{array}
$$

where, $k_{1}$ and $k_{2}$ denote the electron transfer rate constants, $E_{\mathrm{O}_{2} / \mathrm{H}_{2} \mathrm{O}_{2}}^{0}$ and $E_{\mathrm{H}_{2} \mathrm{O}_{2} / \mathrm{H}_{2} \mathrm{O}}^{0}$ the standard potentials of the couples $\mathrm{O}_{2} / \mathrm{H}_{2} \mathrm{O}_{2}$ and $\mathrm{H}_{2} \mathrm{O}_{2} / \mathrm{H}_{2} \mathrm{O}$ respectively. Depending on the electrode surface material and the solution, one or the other pathway, a combination of the two, or even the reaction limited to the oxygen reduction to hydrogen peroxide (2a), may be privileged [26, 28, 29]. In particular, at gold surfaces, the serial pathway seems to be the relevant one in the presence of acidic solutions (e.g. $0.5 \mathrm{M} \mathrm{H}_{2} \mathrm{SO}_{4}$ ) [29, 30]. Nevertheless, the same pathway was observed for gold electrodes in neutral [26] or alkaline solutions [31]. Beyond the $\mathrm{pH}$ value, other determinant parameters are to be considered, including the crystallographic structure of gold as well as the existence and stability of hydroperoxyl $\mathrm{OOH}$ groups at the surface [28]. For the porous electrodes used in this work (see section 4.2), the $\mathrm{pH}$ was shown to be a key parameter. Indeed, experiments carried out at $0.5 \mathrm{M} \mathrm{H} \mathrm{H}_{2} \mathrm{SO}_{4}$ revealed the existence of two distinct waves in the voltammogram which are characteristic for the serial reduction scheme whereas the same experiment, carried out at $0.05 \mathrm{M}$, did not exhibit this feature. Actually, in some configurations, although the potential values of the two couples for this pathway are well separated, the second reaction in (2b) may contribute significantly to the electronic exchange when $\left[\mathrm{H}_{2} \mathrm{O}_{2}\right]$ is not exceedingly small compared to $\left[\mathrm{O}_{2}\right]$, as will be further highlighted in this work. This may occur even at cathodic potentials that are not much smaller than $E_{\mathrm{O}_{2} / \mathrm{H}_{2} \mathrm{O}_{2}}^{0}, \mathrm{O}_{2}$ and $\mathrm{H}_{2} \mathrm{O}_{2}$ being reduced at close potentials [26]. Under 
such circumstances, ignoring the hydrogen peroxide reduction may lead to erroneous interpretation of voltammetry results obtained at potentials that could be thought to be such that the reaction (2b) is negligible a priori. As a result, deriving a formal macroscopic model in which more than one reactive species is involved is of considerable importance. The task is significantly more complex than when only one reaction is involved as the series pathway induces a coupling that needs to be carefully taken into account in the derivation of the macroscopic model. To the best of our knowledge, this has not been reported so far and the present work aims at such a task.

In this work, the series reduction of two species (namely $\mathrm{O}_{2}$ and $\mathrm{H}_{2} \mathrm{O}_{2}$ for further comparison with experiments) is considered together with mass transfer by diffusion yielding a new initial boundary value problem at the pore-scale. Diffusion is supposed to obey Fick's law [32] and the ButlerVolmer formalism is employed to describe the electrochemical kinetics [33]. The pore-scale model is subsequently upscaled using the volume averaging method [34, carrying the coupling between the species to the final diffusionreaction macroscopic model for the series pathway of the oxygen reduction reaction. The associated closure problems, which allow the determination of the macroscopic coefficients (i.e. the effective diffusivities), are provided. Numerical simulations of the initial 3D pore-scale model are employed to successfully validate the 1D macroscopic model. Finally, experimental voltammetry results, obtained with porous gold electrodes in an aqueous acid solution, are compared to the numerical predictions from the macroscopic model, illustrating the performance of the present approach and highlighting the importance of the second reduction reaction (2b) without which fitting the data at low potential fails.

The article is organized as follows. The pore-scale model characterized by diffusion and a serial pathway of two reduction reactions is first developed in section 2. The upscaling procedure to derive the macroscopic model is briefly summarized in section 3, while more details of the derivation are provided in Appendix A. Section 4 is first dedicated to the validation of the upscaled model through comparisons of simulations of its $1 \mathrm{D}$ version with the 3D-DNS of the pore-scale model. Secondly, predictions of the currentto-potential relationship obtained from the macroscopic model are compared to experimental voltammetry data. The improvement of the prediction from the new model including the two reduction reactions developed in the present work with respect to the one reported earlier [13] is clearly highlighted. Concluding remarks are drawn in section 5 . 


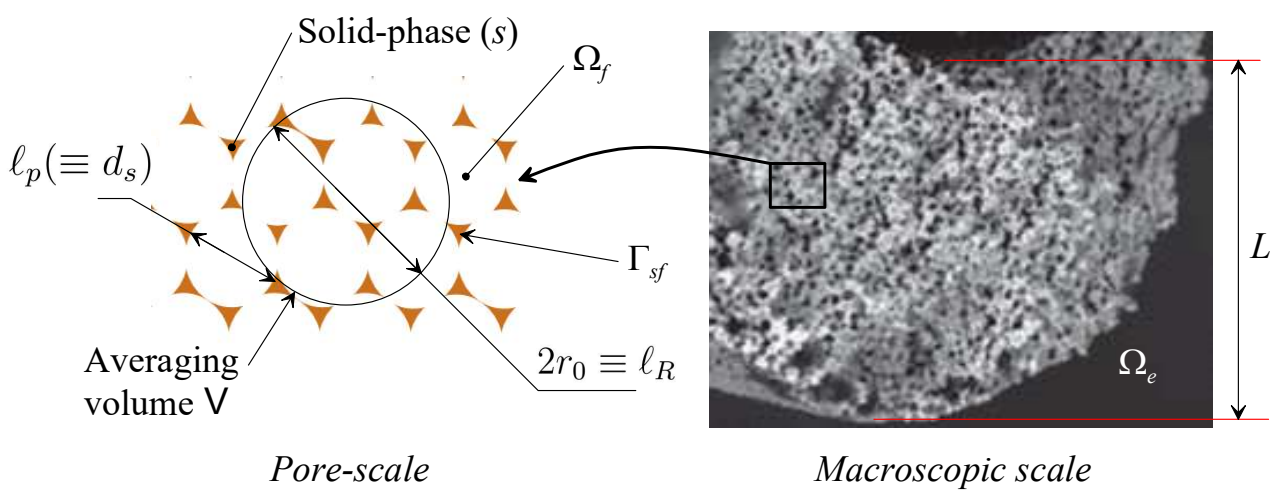

Figure 2.1: Schematic representation of the configuration under study showing the fluid domains inside $\left(\Omega_{f}\right)$ and outside $\left(\Omega_{e}\right)$ the electrode and the characteristic lengths at the pore-scale $\left(\ell_{p}\right)$ and at the macroscopic scale $(L)$. The right picture is a SEM image of a cross section of a cylindrical porous electrode of thickness $L$. $\mathrm{V}$ represents the averaging volume, of radius $r_{0}$, used to upscale the pore-scale problem; $d_{s}$ and $\ell_{R}$ are respectively the size of the spherical pores and the size of the periodic unit cell in the special case of a FCC structure (see section 4).

\section{Pore-scale model}

The system under study is schematically represented in Fig. 2.1, showing the pore and macroscopic scales and the associated characteristic lengths. The development starts with the statement of the microscopic model at the pore-scale involving a pair of serial electrochemical reactions coupled with diffusion of the reactive species within a porous electrode. Let $A$ and $B$ denote these two species (e.g. respectively $\mathrm{O}_{2}$ and $\mathrm{H}_{2} \mathrm{O}_{2}$ in the reaction pathway (2) and $c_{A}$ and $c_{B}$ their molar concentrations in the liquid aqueous solution saturating the pores and in which the electrode is immersed. The reaction rates associated to these two reactions are given by the ButlerVolmer relationship 33 ]

$$
\begin{aligned}
& R_{A}=-k_{1} \alpha_{A} c_{A} \\
& R_{B}=-k_{2} \alpha_{B} c_{B}+k_{1} \alpha_{A} c_{A}
\end{aligned}
$$


with

$$
\begin{aligned}
& \alpha_{A}=\exp \left(\frac{-\alpha_{1} n_{1} F\left(E-E_{O_{2} / H_{2} O_{2}}^{0}\right)}{R T}\right) \\
& \alpha_{B}=\exp \left(\frac{-\alpha_{2} n_{2} F\left(E-E_{H_{2} O_{2} / H_{2} O}^{0}\right)}{R T}\right)
\end{aligned}
$$

where $\alpha_{1}$ and $\alpha_{2}$ denote the electron transfer coefficients, $n_{1}$ and $n_{2}$ the numbers of transferred electrons, for reactions (2a) and (2b) respectively, $R$, $F$ and $T$ being the ideal gas constant, Faraday's constant and the absolute temperature. It should be noted that both reduction reactions in (2) are considered to be irreversible so that only the forward Butler-Volmer's equation is used and the process takes place under isothermal conditions.

The diffusion mechanism of the two species occurring within the porous electrode is considered as a Fickian process. Together with the solid/fluid interface, initial and boundary conditions, this leads to write the governing equations for the transport and reaction of species $i=A, B$ under the form of the following initial boundary value problem

$$
\begin{array}{lr}
\frac{\partial c_{i}}{\partial t}=\boldsymbol{\nabla} \cdot\left(\mathcal{D}_{i} \boldsymbol{\nabla} c_{i}\right) & \text { in } \Omega_{f} \\
-\mathbf{n} \cdot\left(\mathcal{D}_{i} \nabla c_{i}\right)=-R_{i} & \text { at } \Gamma_{s f} \\
c_{i}=\mathcal{F}_{i}(\mathbf{r}) & \mathbf{r} \in \Omega_{f}, \mathrm{t}=0 \\
c_{i}=\mathcal{G}_{i}(\mathbf{r}, t) & \mathbf{r} \in \mathrm{A}_{f \mathrm{e}}, \forall \mathrm{t}
\end{array}
$$

Here $\mathcal{D}_{i}$ denotes the molecular diffusion coefficients of species $i, \mathbf{n}$ the normal unit vector at the solid/fluid interface, $\Gamma_{s f}$, pointing out of the fluid phase. In addition, $\mathrm{A}_{f \mathrm{e}}=\Omega_{f} \cap \Omega_{e}$ is the entrance and/or exit boundary of the fluid phase occupying the domain $\Omega_{f}$ inside the electrode from/into the diffusion layer occupying the region $\Omega_{e}$ outside the electrode, next to it (see Fig. 2.1). It should be noted that in the diffusion layer surrounding the electrode, the diffusion mechanism of both species is governed by Fick's second law given in Eq. (5a). It must be noted that the formal set of assumptions and restrictions for this model to be valid is not easy to identify. However, detailed analyses indicate that if the system is such that species are dilute, the total density and total molar concentration of the mixture are constant, and when the molar fluxes of both species are the same order of magnitude, Eq. (5a) is a reasonable approximation [35, 36]. These restrictions and constraints are retained here. 


\section{Upscaled model}

In this section, our aim is to obtain a macroscopic model by upscaling the above pore-scale problem given in Eqs. (5), using the volume averaging method [34]. The difficulty here lies in the coupled multi-reactions and multidiffusive species problem, yielding a much more complex procedure than the one carried out in [13]. For the sake of conciseness, only the result of the upscaling process is provided below. Details on how to obtain this result are reported in Appendix A. Let $\left\langle c_{i}\right\rangle^{f}(i=A, B)$ represent the intrinsic average concentration in the fluid phase defined by

$$
\left\langle c_{i}\right\rangle^{f}=\frac{1}{V_{f}} \int_{\mathrm{V}_{f}(\mathbf{x})} c_{i} d V
$$

where $\mathrm{V}_{f}$ (of volume $V_{f}$ ) is the region occupied by the fluid phase within the averaging domain. The macroscopic model, coupling the concentration evolution of both species, is given by

$$
\begin{aligned}
& \varepsilon \frac{\partial\left\langle c_{A}\right\rangle^{f}}{\partial t}=\boldsymbol{\nabla} \cdot\left(\varepsilon \mathcal{D}_{A} \mathbf{D}_{\text {eff }}^{*} \cdot \boldsymbol{\nabla}\left\langle c_{A}\right\rangle^{f}\right)-k_{1} \alpha_{A} a_{v}\left\langle c_{A}\right\rangle^{f} \\
& \varepsilon \frac{\partial\left\langle c_{B}\right\rangle^{f}}{\partial t}=\boldsymbol{\nabla} \cdot\left(\varepsilon \mathcal{D}_{B} \mathbf{D}_{e f f}^{*} \cdot \nabla\left\langle c_{B}\right\rangle^{f}\right)+k_{1} \alpha_{A} a_{v}\left\langle c_{A}\right\rangle^{f}-k_{2} \alpha_{B} a_{v}\left\langle c_{B}\right\rangle^{f}
\end{aligned}
$$

where $\varepsilon$ and $a_{v}$ are the porosity and specific area (see their formal definition in Appendix A ) whereas $\mathbf{D}_{\text {eff }}^{*}$ denotes the effective diffusivity tensor given in (A.17a), namely

$$
\mathbf{D}_{e f f}^{*}=\mathbf{I}+\frac{1}{V_{f}} \int_{\mathbf{A}_{s f}} \mathbf{n b} d A
$$


Here, $\mathrm{A}_{s f}$ represents the solid-fluid interface contained in the averaging domain, considered as the Representative Elementary Volume (that will be restricted to a unit cell) of the pseudo periodic structure within which the vector $\mathbf{b}$ is solution of the following intrinsic closure problem (see Eqs. A.12)

$$
\begin{aligned}
\nabla^{2} \mathbf{b} & =0 \quad \text { in } \mathrm{V}_{f} \\
\mathbf{n} \cdot \boldsymbol{\nabla} \mathbf{b} & =-\mathbf{n} \quad \text { at } \mathrm{A}_{s f} \\
\langle\mathbf{b}\rangle^{f} & =0 \\
\mathbf{b}(\mathbf{r}) & =\mathbf{b}\left(\mathbf{r}+\ell_{i} \mathbf{e}_{i}\right) \quad i=1,2,3
\end{aligned}
$$

Here, $\ell_{i} \mathbf{e}_{i}(i=1,2,3)$ are the periodic lattice vectors of the unit cell. When complemented with the initial and boundary conditions for $\left\langle c_{A}\right\rangle^{f}$ and $\left\langle c_{B}\right\rangle^{f}$, Eqs. (8) form the effective macroscopic model for the coupled diffusion and serial reduction reactions of species $A$ and $B$. It must be emphasized that its validity is subject to three constraints. The first one is on the lenght-scales, i.e., $\ell_{p} \ll L\left(\ell_{p}\right.$ is the characteristic pore-size and $L$ the macroscopic lengthscale of the medium, see Fig. 2.1). The second one, on the kinetic number expressed as $K i=\ell_{p} \max \left(k_{1} \alpha_{A} / \mathcal{D}_{A}, k_{2} \alpha_{B} / \mathcal{D}_{B}\right)$, is given by $K i \ll 1$. The last one is on the time scale at which the process is observed which must be such that $t / \ell_{p}^{2} \min \left(\mathcal{D}_{A}, \mathcal{D}_{B}\right) \gg 1$ [34, 13]. The macroscopic concentration fields of $\left\langle c_{i}\right\rangle^{f}(i=A, B)$, solution of the macroscopic model, allows the determination of the current available at the electrode which takes the form

$$
I=-n_{1} k_{1} F \alpha_{A} a_{v} \int_{\Omega}\left\langle c_{A}\right\rangle^{f} \mathrm{~d} V-n_{2} k_{2} F \alpha_{B} a_{v} \int_{\Omega}\left\langle c_{B}\right\rangle^{f} \mathrm{~d} V
$$

$\Omega$ representing the entire domain occupied by the electrode.

\section{Numerical results}

In this section, the macroscopic model is first validated from comparisons with pore-scale DNS on a given model structure. Predictions of the current delivered by an electrode during voltammetry tests from the macroscopic model are further compared to experimental data.

\subsection{Pore-scale model DNS and numerical solutions of the macroscopic model}

To carry out 3D DNS of the pore-scale model (Eqs. (5p) and compare the results with those obtained from the 1D solution of the macroscopic model derived in section 3, a model configuration is considered. It is represented in 


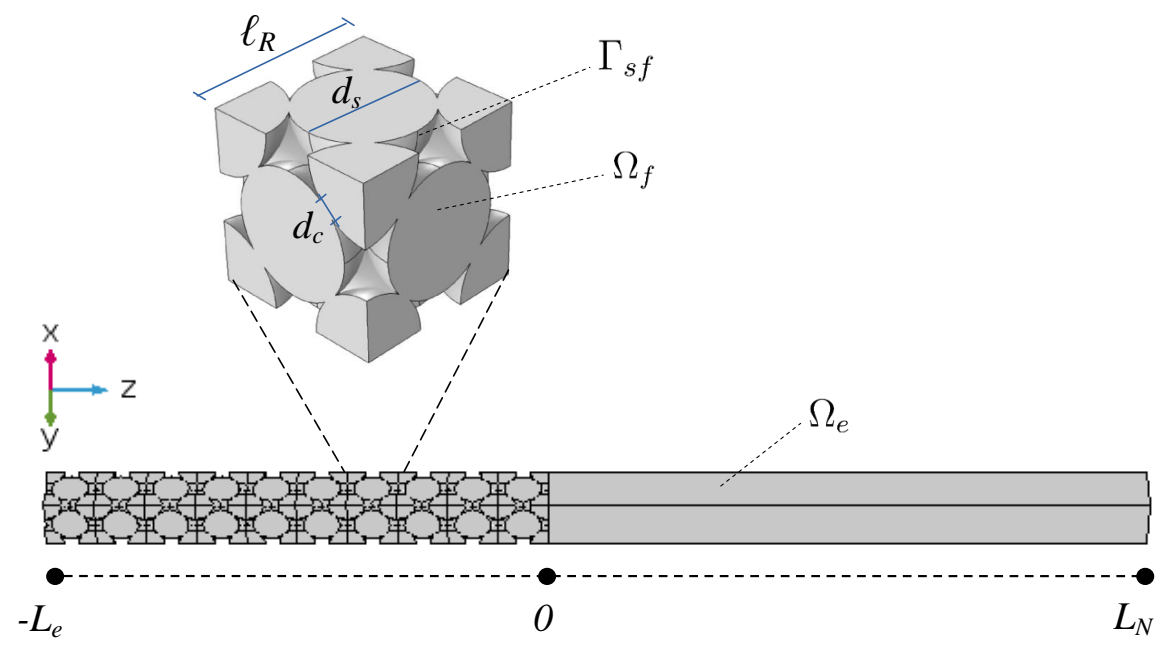

Figure 4.1: 3D domain for DNS and unit cell of the porous structure. All fluid domains are represented in gray; the solid phase is not depicted

Fig. 4.1 where the computational domain for the DNS and the periodic unit cell, of size $\ell_{R}$, of the porous structure are reported. The electrode, of thickness $L_{e}$, is supposed to be composed of a face-centered-cubic (FCC) arrangement of spherical pores of diameter $d_{s}=\ell_{p}$ connected to each other through a circular window of diameter $d_{c}$. Its extensions in the $x$ - and $y$-directions are supposed to be large enough for periodicity to be a valid approximation in both directions. For this approximation to hold, it is first assumed that the electrode length is much larger than $\ell_{R}$. Second, the electrode is supposed to be either a plane one, with a very large lateral extension compared to $\ell_{R}$, or is circular with a thickness $L_{e}$ much smaller than its mean radius. As a consequence, the 3D computational domain for the pore-scale DNS is restricted to a single unit cell in the $x$ - and $y$-directions (see Fig. 4.1).

The electrode, positioned between $z=-L_{e}$ and $z=0$, lies on its solid impermeable and electrically conducting support at $z=-L_{e}$ so that a zero diffusive flux for both species is applied at this location. Moreover, the electrode is in contact with the bulk solution at $z=0$ where a Nernstian diffusion layer, between $z=0$ and $z=L_{N}$, settles down. At the free extremity of the diffusion layer, a Dirichlet boundary condition is applied for the two species, i.e. $c_{i}=c_{i}^{0}, i=A, B$ at $z=L_{N}$. Finally, periodic boundary conditions on $c_{i}, i=A, B$ (and their gradients) are applied in the $x$ - and $y$-directions. The initial concentrations are supposed to be uniform, equal to $c_{i}^{0}, i=A, B$. 
The system of pore-scale equations (5) is made dimensionless using $\ell_{R}$, $\ell_{R}^{2} / D_{A}$ and $c_{A}^{0}$ as the reference quantities for length, time and concentrations respectively. Denoting dimensionless quantities with the superscript ${ }^{*}$, this yields the following initial boundary value problem to be solved

$$
\begin{aligned}
& \frac{\partial c_{A}^{*}}{\partial t^{*}}=\nabla^{* 2} c_{A}^{*}, \quad \text { in } \Omega_{f} \cup \Omega_{e} \\
& \frac{\partial c_{B}^{*}}{\partial t^{*}}=\frac{\mathcal{D}_{B}}{\mathcal{D}_{A}} \nabla^{* 2} c_{B}^{*}, \quad \text { in } \Omega_{f} \cup \Omega_{e} \\
& \text { B.C.1 } \quad-\mathbf{n} \cdot \nabla^{*} c_{A}^{*}=\frac{\ell_{R}}{\mathcal{D}_{A}} k_{1} \alpha_{A} c_{A}^{*} \quad \text { at } \Gamma_{s f} \\
& \text { B.C.2 }-\mathbf{n} \cdot \nabla^{*} c_{B}^{*}=\frac{\ell_{R}}{\mathcal{D}_{B}}\left(k_{2} \alpha_{B} c_{B}^{*}-k_{1} \alpha_{A} c_{A}^{*}\right) \quad \text { at } \Gamma_{s f} \\
& \text { B.C.3 } \quad-\mathbf{n} \cdot \nabla^{*} c_{i}^{*}=0, \quad i=A, B \quad \text { at } z^{*}=-L_{e}^{*} \\
& \text { B.C.4 } c_{A}^{*}=1 \quad \text { at } z^{*}=L_{N}^{*} \\
& \text { B.C.5 } c_{B}^{*}=c_{B}^{0} / c_{A}^{0} \quad \text { at } z^{*}=L_{N}^{*} \\
& \text { I.C.1 } c_{A}^{*}=1 \quad \text { in } \Omega_{f} \cup \Omega_{e} \text { at } t^{*}=0 \\
& \text { I.C.2 } \quad c_{B}^{*}=c_{B}^{0} / c_{A}^{0} \quad \text { in } \Omega_{f} \cup \Omega_{e} \text { at } t^{*}=0 \\
& c_{i}^{*}\left(x^{*}, y^{*}, z^{*}\right)=c_{i}^{*}\left(x^{*}+1, y^{*}+1, z^{*}\right), \quad i=A, B \quad \text { in } \Omega_{f} \\
& \mathbf{n} \cdot \nabla^{*} c_{i}^{*}\left(x^{*}, y^{*}, z^{*}\right)=\mathbf{n} \cdot \nabla^{*} c_{i}^{*}\left(x^{*}+1, y^{*}+1, z^{*}\right), \quad i=A, B \quad \text { in } \Omega_{f}
\end{aligned}
$$

The software COMSOL Multiphysics (version 5.2a) was used to solve this $3 \mathrm{D}$ problem. Careful attention was paid to the mesh convergence and we used a physics-controlled mesh including extremely fine grid blocks composed of $9 \times 10^{6}$ tetrahedral elements in the overall domain represented in Fig. 4.1.

The 1D macroscopic model was also solved in the same conditions. In that case, the computational domain reduces to the two sub-domains $\Omega$ and $\Omega_{e}$ as depicted in Fig. 4.2. At the boundary between the electrode and the diffusion

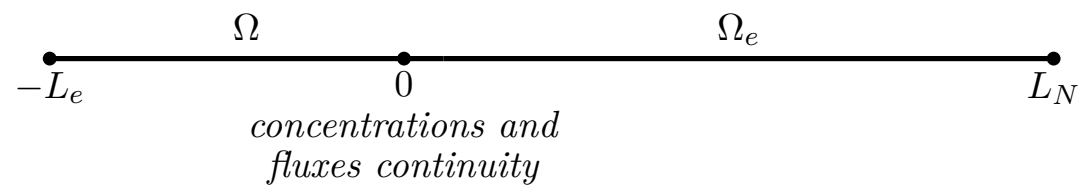

Figure 4.2: 1D reduced domain for the simulation of the macroscopic model layer, i.e. at $z=0$, continuity of both the concentrations and diffusive fluxes 
of each species is considered. This choice for the boundary conditions at the macroscopic porous-fluid interface was shown to be an appropriate one in the framework of the underlying hypotheses for the problem under consideration [13. Using the same reference quantities, the dimensionless form of the macroscopic problem to be solved can be stated as follows

$$
\begin{aligned}
& \frac{\partial\left\langle c_{A}^{*}\right\rangle^{f}}{\partial t^{*}}=\mathrm{D}_{e f f}^{*} \frac{\partial^{2}\left\langle c_{A}^{*}\right\rangle^{f}}{\partial z^{* 2}}-\frac{a_{v} \ell_{R}^{2}}{\varepsilon \mathcal{D}_{A}} k_{1} \alpha_{A}\left\langle c_{A}^{*}\right\rangle^{f} \quad \text { in } \Omega \\
& \frac{\partial\left\langle c_{B}^{*}\right\rangle^{f}}{\partial t^{*}}=\frac{\mathcal{D}_{B}}{\mathcal{D}_{A}} \mathrm{D}_{e f f}^{*} \frac{\partial^{2}\left\langle c_{B}^{*}\right\rangle^{f}}{\partial z^{* 2}}+\frac{a_{v} \ell_{R}^{2}}{\varepsilon \mathcal{D}_{A}}\left(k_{1} \alpha_{A}\left\langle c_{A}^{*}\right\rangle^{f}-k_{2} \alpha_{B}\left\langle c_{B}^{*}\right\rangle^{f}\right) \quad \text { in } \Omega \\
& \text { B.C.1 }\left\langle c_{i}^{*}\right\rangle^{f}=c_{i}^{*}, \quad i=A, B \quad \text { at } z^{*}=0 \\
& \text { B.C.2 } \varepsilon \mathrm{D}_{\text {eff }}^{*} \frac{\partial\left\langle c_{i}^{*}\right\rangle^{f}}{\partial z^{*}}=\frac{\partial c_{i}^{*}}{\partial z^{*}}, \quad i=A, B \quad \text { at } z^{*}=0 \\
& \frac{\partial c_{A}^{*}}{\partial t^{*}}=\frac{\partial^{2} c_{A}^{*}}{\partial z^{* 2}}, \quad \text { in } \Omega_{e} \\
& \frac{\partial c_{B}^{*}}{\partial t^{*}}=\frac{\mathcal{D}_{B}}{\mathcal{D}_{A}} \frac{\partial^{2} c_{B}^{*}}{\partial z^{* 2}}, \quad \text { in } \Omega_{e} \\
& \text { B.C.3 } \frac{\partial\left\langle c_{i}^{*}\right\rangle^{f}}{\partial z^{*}}=0, \quad i=A, B \quad \text { at } z^{*}=-L_{e}^{*} \\
& \text { B.C. } 4 \quad c_{A}^{*}=1 \quad \text { at } z^{*}=L_{N}^{*} \\
& \text { B.C.5 } c_{B}^{*}=\frac{c_{B}^{0}}{c_{A}^{0}} \quad \text { at } z^{*}=L_{N}^{*} \\
& \text { I.C. } 1\left\langle c_{A}^{*}\right\rangle^{f}=1 \quad \text { in } \Omega \text { at } t^{*}=0 \\
& \text { I.C. } 2\left\langle c_{B}^{*}\right\rangle^{f}=\frac{c_{B}^{0}}{c_{A}^{0}} \quad \text { in } \Omega \text { at } t^{*}=0 \\
& \text { I.C. } 3 \quad c_{A}^{*}=1 \text { in } \Omega_{e} \text { at } t^{*}=0 \\
& \text { I.C. } 4 \quad c_{B}^{*}=\frac{c_{B}^{0}}{c_{A}^{0}} \quad \text { in } \Omega_{e} \text { at } t^{*}=0
\end{aligned}
$$

In the above equations, $D_{\text {eff }}^{*}$ is such that, due to isotropy of the unit cell,

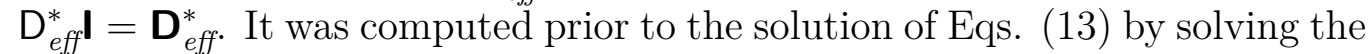
closure problem in Eqs. (10). Both problems were also solved with COMSOL Multiphysics.

The micro- and macroscale simulations correspond to a voltammetry numerical experiment carried out with a scanning potential ranging from $E=0.4 \mathrm{~V}$ to $0 \mathrm{~V}$ at a constant scan rate $r_{E}=5 \mathrm{mV} / \mathrm{s}$. Parameters used 


\begin{tabular}{cccc} 
Parameter & Symbol & Value & Unit \\
\hline Ideal gas constant & $R$ & 8.314 & $\mathrm{~J} /(\mathrm{molK})$ \\
Faraday's constant & $F$ & 96485 & $\mathrm{C} / \mathrm{mol}$ \\
Temperature & $T$ & 298 & $\mathrm{~K}$ \\
Number of transferred electrons & $n_{1}, n_{2}$ & 2 & - \\
Electron transfer coefficient & $\alpha_{1}$ & 0.58 & - \\
Electron transfer coefficient & $\alpha_{2}$ & 0.482 & - \\
Electron transfer rate constant & $k_{1}$ & $1.210^{-7}$ & $\mathrm{~cm} / \mathrm{s}$ \\
Electron transfer rate constant & $k_{2}$ & $1.710^{-17}$ & $\mathrm{~cm} / \mathrm{s}$ \\
Standard potential vs. $E_{A g / A g C l}^{0}$ & $E_{\mathrm{O}_{2}}^{0}$ & 0.45 & $\mathrm{~V}$ \\
Standard potential vs. $E_{\mathrm{Ag} / \mathrm{AgCl}}^{0}$ & $E_{\mathrm{H}_{2} \mathrm{O}_{2}}^{0}$ & 1.56 & $\mathrm{~V}$ \\
Initial and bulk concentration of $\mathrm{O}_{2}$ & $c_{A}^{0}$ & 1.2 & $\mathrm{~mol} / \mathrm{m}^{3}$ \\
Initial and bulk concentration of $\mathrm{H}_{2} \mathrm{O}_{2}$ & $c_{B}^{0}$ & 0 & $\mathrm{~mol} / \mathrm{m}^{3}$ \\
Diffusion coefficient of $\mathrm{O}_{2}$ & $D_{A}[37]$ & $1.910^{-9}$ & $\mathrm{~m} / \mathrm{s}$ \\
Diffusion coefficient of $\mathrm{H}_{2} \mathrm{O}_{2}$ & $D_{B}[37]$ & $1.410^{-9}$ & $\mathrm{~m} / \mathrm{s}$ \\
Dimensionless effective diffusivity & $\mathrm{D}_{e f f}^{*}$ & 0.493 & - \\
Spherical pore diameter & $d_{s}$ & 1.2 & $\mathrm{\mu m}$ \\
Connecting window-size & $d_{c}$ & $0.15 \mathrm{~d}_{s}$ & $\mathrm{\mu m}$ \\
Size of the periodic unit cell & $\ell_{R}$ & 1.678 & $\mu \mathrm{m}$ \\
Porosity & $\varepsilon$ & 0.763 & \\
Specific area & $a_{v}$ & $3.567 \times 10^{6}$ & $1 / \mathrm{m}$ \\
\hline
\end{tabular}

Table 4.1: Parameters used in the simulations

in the simulations are provided in Table. 4.1. The electrode thickness was chosen to be $L_{e}=10 \ell_{R}$ which corresponds to 40 half layers (HL) of spherical pores. The diffusion layer thickness, $L_{N}$, was taken equal to $30 \mu m$. It must be noted that, because of a pseudo-elementary rate determining step, a single electron transfer is to be considered [38], even though both reduction reactions occur with two electrons [39]. As a consequence, the values of $n_{1}$ and $n_{2}$ were taken equal to 1 in the exponential term of the driving force in the Butler-Volmer relationships (4).

In Fig. 4.3 the $\mathrm{O}_{2}$ concentration profiles within the electrode during voltammetry obtained from the solution of the 1D macroscopic model (Eqs. (13p) and 3D DNS of the pore-scale model (Eqs. (12p) are represented at three different times. Clearly, the agreement between the two approaches is 


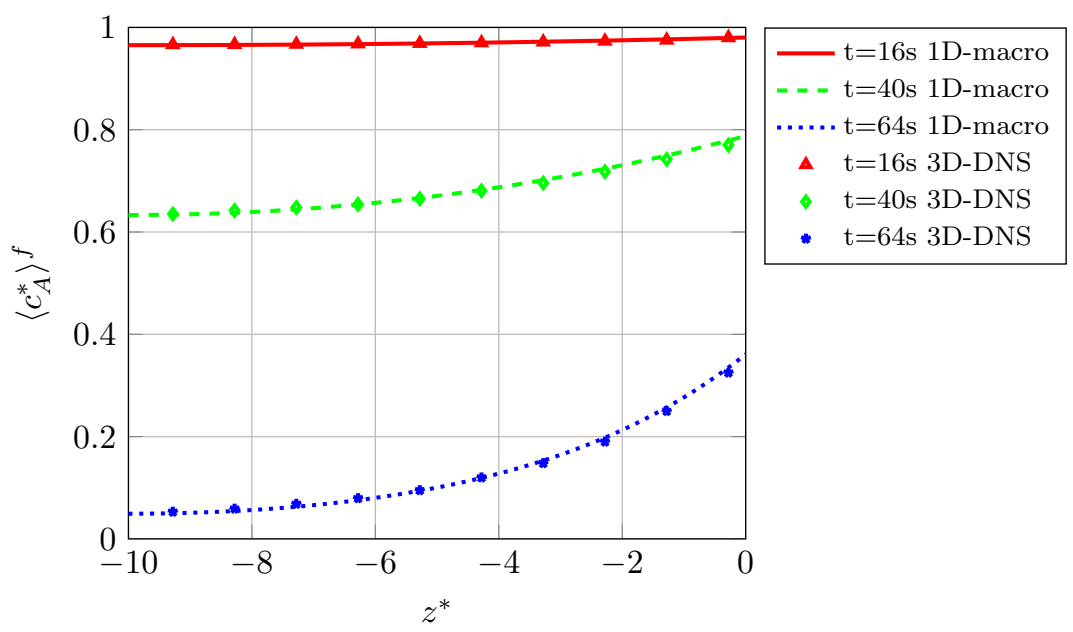

Figure 4.3: Dimensionless $\mathrm{O}_{2}$ concentration profiles within the porous electrode obtained from the solution of the 1D macroscopic model and from 3D DNS of the pore-scale model

excellent. This is further confirmed in Fig. 4.4 in which evolutions of the dimensionless mean concentrations, $\overline{c_{i}^{*}}, i=A, B$, over the entire electrode domain are represented. The mean concentrations are given by the averages $\frac{1}{|\Omega|} \int_{\Omega}\left\langle c_{i}^{*}\right\rangle^{f} \mathrm{~d} V$ and $\frac{1}{\left|\Omega_{f}\right|} \int_{\Omega_{f}} c_{i}^{*} \mathrm{~d} V$, for the $1 \mathrm{D}$ macroscopic approach and $3 \mathrm{D}$ DNS at the pore-scale respectively, $|\Omega|$ and $\left|\Omega_{f}\right|$ denoting the volume of $\Omega$ and $\Omega_{f}$. Again, results obtained from the pore-scale DNS and from the solution of the macroscopic model match perfectly.

For completeness, a final comparison is made on the current delivered by the electrode versus the applied potential obtained from the macroscopic and pore-scale approaches, the current, $I$, being computed from Eqs. (11) and (6) respectively. The voltammogram is represented in Fig. 4.5 showing again the excellent agreement between the two results. These very successful comparisons prove the validity of the new upscaled model.

The net advantage of the macroscopic approach lies in the gain in terms of computational resources and time required to obtain the (average) concentration fields and the current. In fact, for the case under study, the computational time is only 9s for the $1 \mathrm{D}$ macroscopic model while 108 minutes are needed for a 3D DNS of the pore-scale model (on a Dell PowerEdge 430-2 processors Intel Xeon E5-2630v3), leading to a speed-up of about 720. In 


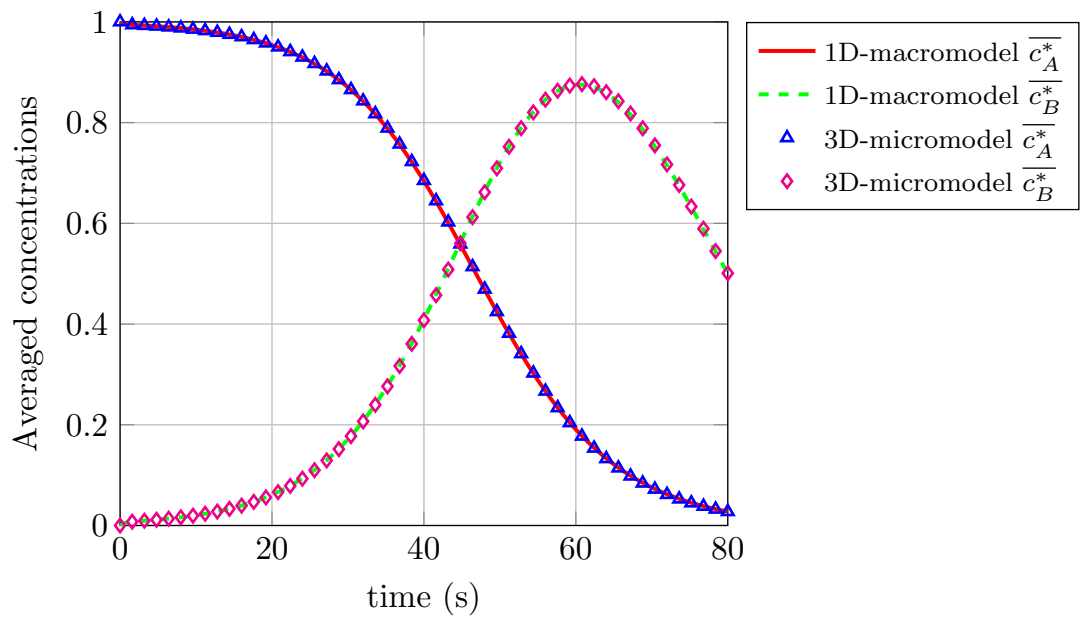

Figure 4.4: Dimensionless mean concentrations of $\mathrm{O}_{2}$ and $\mathrm{H}_{2} \mathrm{O}_{2}$ within the porous electrode obtained from the simulation of the $1 \mathrm{D}$ macroscopic model and from 3D DNS of the pore-scale model

addition, the upscaling procedure provides a comprehensive model operating at the macroscopic scale and the link with the microscale parameters.

\subsection{Comparison with experimental data}

The aim of this section is to test the ability of the new macroscopic model developed here to accurately predict the current-to-potential characteristics obtained from voltammetry experiments carried out with porous electrodes for the serial oxygen reduction reactions to water as described by (2). Three porous electrodes were prepared according to a protocol that has been widely described in the literature [40, 41, 42, 43]. This protocol mainly consists of three steps: i) deposition of layers of organized colloidal self-assembled silica beads is first performed on a gold wire of $250 \mu \mathrm{m}$ in diameter using the Langmuir-Blodgett method. Multiple silica particle layers were obtained by repeating the dipping and withdrawing $(1.2 \mathrm{~mm} / \mathrm{min})$ process. Spherical silica beads of $1.2 \mu \mathrm{m}$ in diameter were employed here; ii) filling the bead assembly with gold is carried out by electrodeposition (Elevate $\AA$ Gold 7990, $-0.6 \mathrm{~V})$; iii) beads are dissolved with hydrofluoric acid, leaving an electrically conducting porous structure which pore diameter, $d_{s}\left(=\ell_{p}\right)$, corresponds to the bead diameter. Since gold electrodeposition does not lead to a complete filling of the gaps between the beads, connecting windows between the 


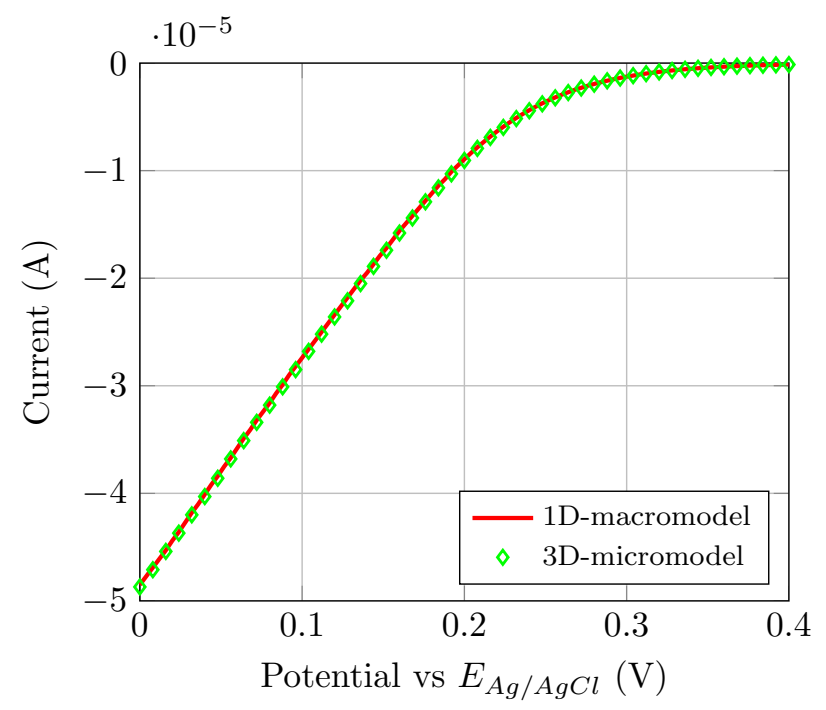

Figure 4.5: Current available at the electrode versus the applied scanning potential computed with the 1D macroscopic (Eq. (11)) and 3D microscopic (Eq. (60) models

spherical pores remain, yielding an inverse opal-like percolating porous structure. The connecting window-size, $d_{c}$, was characterized to be approximately $0.15 d_{s}$. As a result of the self-assembling mechanism, the structure is very compact and can reasonably be represented by an FCC structure (see Fig. 4.1). The three electrodes are made of 5, 11 and 19 half-layers of spherical pores respectively (referred to as 5HL, 11HL and 19HL in the following). It should be noted that a $4 \mathrm{HL}$ structure corresponds to $1 \mathrm{FCC}$ unit cell for modelling.

The electrodes were immersed in a $0.5 \mathrm{M} \mathrm{H}_{2} \mathrm{SO}_{4}$ solution saturated by oxygen in order to carry out voltammetry experiments at a constant temperature $T=298 \mathrm{~K}$ and a constant scan rate of $5 \mathrm{mV} / \mathrm{s}$ with a decreasing potential in the range from $0.4 \mathrm{~V}$ to $0 \mathrm{~V}$. All the electrochemical measurements were carried out with an Autolab PGSTAT101 potentiostat. A cylindrical flexible carbon sheet was used as counter electrode, and $\mathrm{Ag} / \mathrm{AgCl}(3 \mathrm{M} \mathrm{KCl})$ was used as a reference electrode. Electrochemical measurements were carried out inside a sealed cell, the $0.5 \mathrm{M} \mathrm{H}_{2} \mathrm{SO}_{4}$ solution was always purged with $\mathrm{O}_{2}$ for $15 \mathrm{~min}$ before the measurement, and a slow $\mathrm{O}_{2}$ flow was kept above the $0.5 \mathrm{M} \mathrm{H}_{2} \mathrm{SO}_{4}$ solution in order to maintain the constant $\mathrm{O}_{2}$ concentration. 


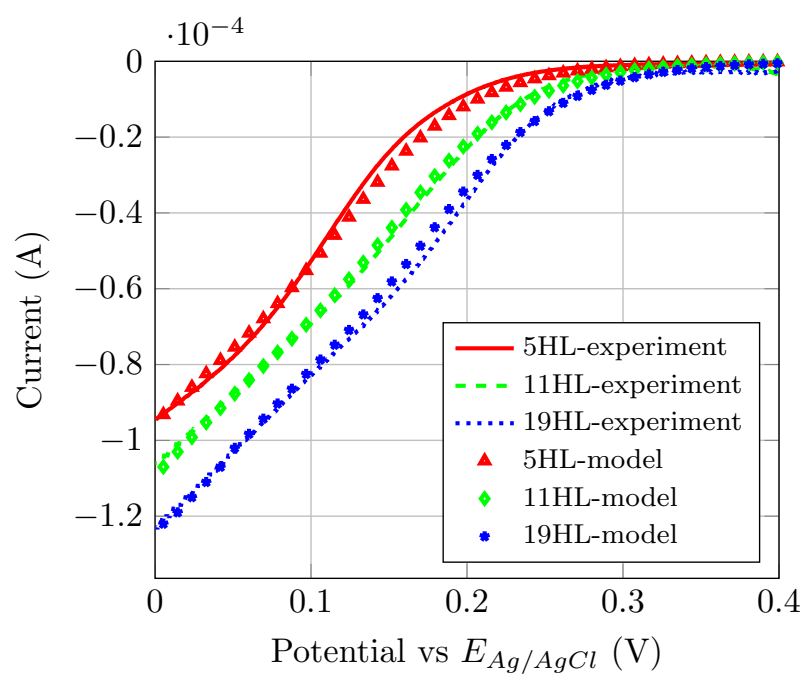

Figure 4.6: Experimental voltammogram obtained with the three electrodes in a $0.5 \mathrm{M} \mathrm{H}_{2} \mathrm{SO}_{4}$ solution saturated with oxygen at a $5 \mathrm{mV} / \mathrm{s}$ scan rate. Comparison with predictions obtained from the macroscopic model in Eqs. (13)

Experimental results obtained with the three electrodes are reported in Fig. 4.6. It should be noted that all the experiments were repeated at least three times to check repeatability.

The electron transfer rate constants, $k_{1}$ and $k_{2}$, and electron transfer coefficients, $\alpha_{1}$ and $\alpha_{2}$, together with the diffusion layer thickness, $L_{N}$, are not accurately known a priori. For this reason, these parameters were fitted in the macroscopic 1D model given in Eqs. (13) and the current expressed in Eq. (11) in the least square sense. This was performed on the curve obtained with the 11HL electrode and optimal values for $k_{1}, k_{2}, \alpha_{1}$ and $\alpha_{2}$ (together with all the other required data for the solution of the macroscopic model) are reported in Table 4.1. The diffusion layer thickness yielding the best fit was found to be $L_{N}=130 \mu m$. Values of the parameters fitted on the voltammogram obtained with the 11HL electrode were then kept the same for the simulations of the 1D macroscopic model for the 5HL and 19HL electrodes. Numerical results are reported in Fig. 4.6 as a comparison with experimental data, showing an excellent agreement. In fact, as reported in Fig. 4.7, the absolute value of the relative error on the current over the whole range of scanning potential is less than $5 \%$ for all the electrodes. This 


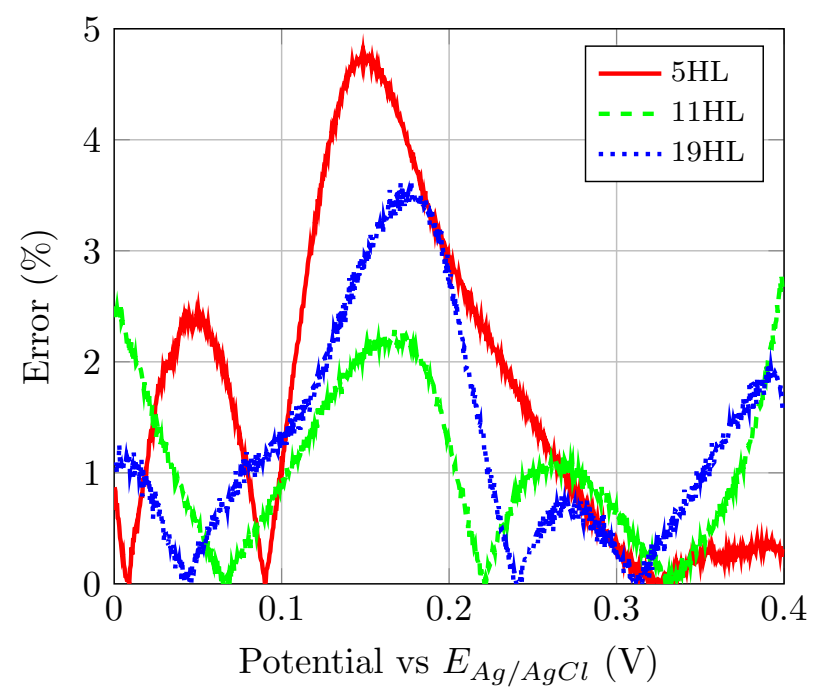

Figure 4.7: Absolute value of the relative error on the current between simulation results and experimental data. The experimental data set of Fig. 4.6 for the 11HL electrode was employed to fit the parameters (see text) which were used to perform simulations of the 1D macroscopic model for the 5HL and 19HL electrodes

demonstrates the ability and the accuracy of the new macroscopic model derived in this work to predict the behavior of a porous electrode in the case of a serial reaction pathway. In order to appreciate the role of the reduction reaction (2b) of $\mathrm{H}_{2} \mathrm{O}_{2}$ to water that is often neglected, a onereaction version of the macroscopic model was solved, taking into account only the reduction reaction (2a) of $\mathrm{O}_{2}$ to $\mathrm{H}_{2} \mathrm{O}_{2}$, as in [13]. Parameters for the solutions of this reduced model were kept the same as those employed with the full model for the complete serial pathway. Current versus the scanning potential results are reported in Fig. 4.8. From this figure, it can be observed that the reduced model correctly predicts the current in the upper range of the potential values. However, for potentials smaller than $0.2 \mathrm{~V}$ to $0.1 \mathrm{~V}$, depending on the electrode thickness, the second reaction plays obviously a key role in the electronic exchange. This can be explained by the fact that, at the early stage of the experiments, i.e. at large values of the potential, $\left[\mathrm{H}_{2} \mathrm{O}_{2}\right]$ is not significant since the amount of oxygen that has already reacted remains small. More quantitatively, the contribution of the second reduction reaction to the overall current production can be estimated from the analysis 


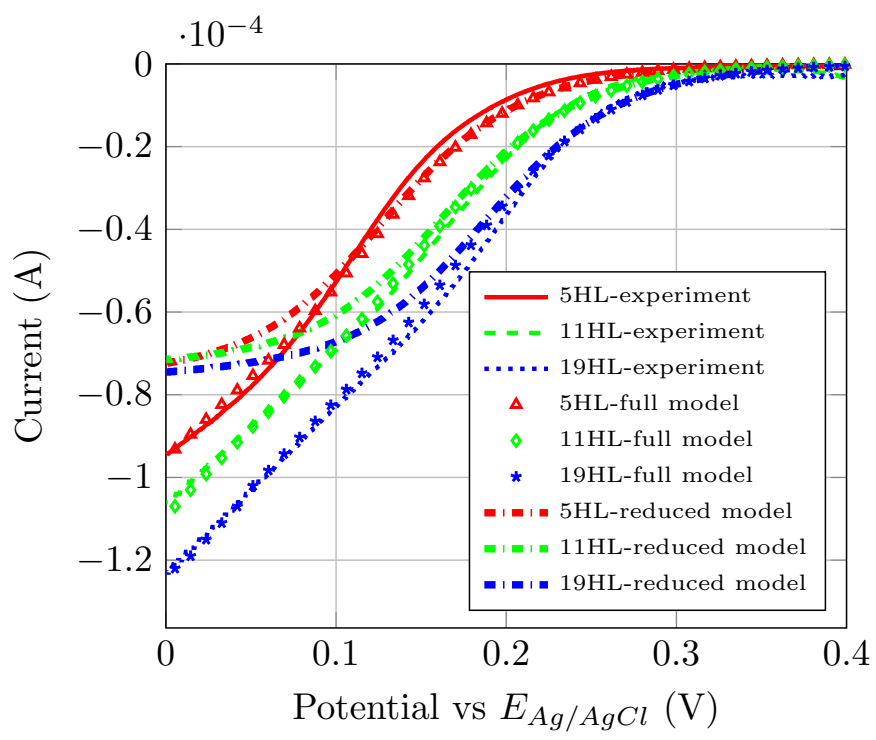

Figure 4.8: Comparison of the voltammetry curves obtained by simulations of the reduced model considering a single reduction reaction $\mathrm{O}_{2}$ to $\mathrm{H}_{2} \mathrm{O}_{2}$ and the full coupled model of the complete serial pathway

of the ratio between the two terms in the right hand side of Eq. (11), which is simply given by $k_{2} \alpha_{B} \overline{c_{B}^{*}} /\left(k_{1} \alpha_{A} \overline{c_{A}^{*}}\right)$. This ratio is represented versus the scanning potential in Fig. 4.9. It can be clearly seen that around $10 \%$ of the total current delivered by the electrode is produced by the second reaction for potentials smaller than $0.1 \mathrm{~V}, 0.14 \mathrm{~V}$ and $0.17 \mathrm{~V}$ for the $5 \mathrm{HL}, 11 \mathrm{HL}$ and 19HL electrodes respectively. This is perfectly consistent with the values of the threshold potentials in Fig. 4.8 at which the reduced model fails to accurately predict the current observed experimentally. This threshold value increases with the electrode thickness and this is due to the fact that, while decreasing the potential, $\left[\mathrm{H}_{2} \mathrm{O}_{2}\right]$ is larger for a thicker electrode as a result of a larger reacting surface. This behavior is further confirmed by the fact that the contribution of the second reduction reaction becomes larger as the electrode thickness increases. For instance, at $0 \mathrm{~V}$, the second reaction contributes to around $33 \%, 45 \%$ and $58 \%$ of the total current produced with the $5 \mathrm{HL}, 11 \mathrm{HL}$ and $19 \mathrm{HL}$ electrodes respectively. In the latter case, the second reaction is even the dominant one. This clearly highlights that this second reaction must not be omitted in the interpretation of the electrochemical behavior of an electrode during oxygen reduction in an aqueous solution. 


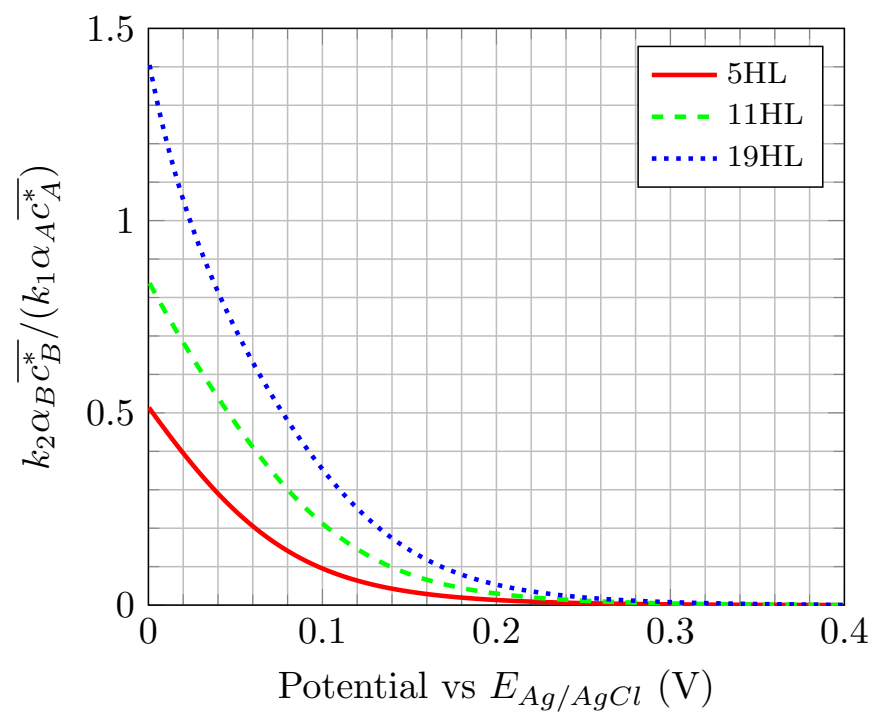

Figure 4.9: Ratio of the current contribution from each of the two serial reduction reactions versus the scanning potential

\section{Conclusions}

In this article, a physico-electrochemical multiscale model of two reduction reactions within a porous electrode via a serial pathway including the diffusion of the two reactive species was developed. The initial boundary value problem at the pore-scale was stated and upscaled to formally derive a new macroscopic model using the volume averaging method. It provides an accurate prediction of the coupled evolution of both molar species concentrations. The associated closure problem allowing the determination of the effective coefficients (more particularly the effective diffusivity) appearing in this model was provided. A validation of the macroscopic model was carried out with a thorough comparison with the results obtained from Direct Numerical Simulations of the pore-scale model in the case of the serial pathway of oxygen reduction to water, clearly assessing the accuracy of the macroscopic model. A very significant computational speed-up is achieved with the macroscopic model.

Voltammetry experiments were carried out on three different porous gold electrodes of different thicknesses immersed in an aqueous acid solution. The macroscopic model was used to predict the current-to-potential relationship after parameters such as the electron transfer rate constants, the electron 
transfer coefficients of both reactions and the diffusion layer thickness have been fitted on the experimental data of only one of the three electrodes. The agreement between the experimental results and the prediction from the model is excellent, showing less than $5 \%$ of error over the whole range of scanning potential and proving the relevance of the macroscopic model. Moreover, simulations showed the importance of the second reduction reaction of hydrogen peroxide to water. It indicates that this reaction very significantly contributes to the overall current available at the electrode when the potential becomes smaller than $0.2 \mathrm{~V}$ to $0.1 \mathrm{~V}$, depending on the electrode thickness. On the basis of its performance, the macroscopic model derived here opens interesting perspectives in terms of the optimization of sophisticated electrode architectures. More generally, this model maybe of major interest in chemical engineering applications, far beyond the context of the present work, whenever a series of heterogeneous reactions is to be considered involving more than one diffusing dilute species. A generalization to reactions involving $n$ species $(n>2)$ may also be envisaged following the same approach to obtain a macroscopic description.

\section{Acknowledgement}

This work was supported by the LabEx AMADEus (ANR-10-LABX42) within IdEx Bordeaux (ANR-10-IDEX-03-02), i.e. the "Investissements d'Avenir Programme" of the French government managed by the Agence Nationale de la Recherche (ANR). It also benefited from the support of the ANR projects MOMA (ANR-17-CE08-0005) and BIO3 (ANR-16-CE19-0001).

\section{Appendix A Volume averaging and derivation of the upscaled model}

In this Appendix, the upscaling procedure to derive the macroscopic diffusion/reaction equations by making use of the volume averaging method is presented. The development is similar to the one described in [34] with an extension to a more complex problem where multiple species and reactions are involved.

The upscaling is carried out by means of the superficial and intrinsic volume averaging operators over a volume $\mathrm{V}$ (of measure $V$ and radius $r_{0}$ ) (see Fig. 2.1) embedding a domain occupied by the fluid phase $V_{f}$ (of measure 
$V_{f}$ ) and the solid-fluid interface $\mathrm{A}_{s f}$ (of measure $A_{s f}$ ). $\mathrm{V}_{f}$ and $\mathrm{A}_{s f}$ are the restrictions of $\Omega_{f}$ and $\Gamma_{s f}$ to $\mathrm{V}$ and $r_{0} \ll L$. The two operators are respectively defined as

$$
\begin{aligned}
\left\langle c_{i}\right\rangle & =\frac{1}{V} \int_{\mathrm{V}_{f}} c_{i} d V \quad i=A, B \\
\left\langle c_{i}\right\rangle^{f} & =\frac{1}{V_{f}} \int_{\mathrm{V}_{f}} c_{i} d V=\varepsilon^{-1}\left\langle c_{i}\right\rangle \quad i=A, B
\end{aligned}
$$

Here, $\varepsilon$ represents the porosity, $\varepsilon=\frac{V_{f}}{V}$. Permutation of the average and the time and space derivative requires the use of the Reynolds transport and averaging theorems which can be respectively expressed as follows [44, 45],

$$
\begin{aligned}
\left\langle\frac{\partial c_{i}}{\partial t}\right\rangle & =\frac{\partial\left\langle c_{i}\right\rangle}{\partial t} \\
\left\langle\boldsymbol{\nabla} c_{i}\right\rangle & =\boldsymbol{\nabla}\left\langle c_{i}\right\rangle+\frac{1}{V} \int_{\boldsymbol{A}_{s f}} \mathbf{n} c_{i} d A
\end{aligned}
$$

and a straightforward form for the latter for the divergence operator. It should be noticed that while writing Eq. A.2a), $V$ was assumed to be independent of time due to a non-deformable porous medium.

The superficial average operator is applied to the microscale equations (5a) and, after making use of the interfacial boundary conditions (5b) together with the hypothesis that the pore and macroscopic characteristic length-scales $\ell_{p}$ and $L$ are well separated, i.e. $\ell_{p} \ll L$, this yields

$$
\begin{aligned}
\varepsilon \frac{\partial\left\langle c_{A}\right\rangle^{f}}{\partial t}= & \boldsymbol{\nabla} \cdot\left[\mathcal{D}_{A}\left(\varepsilon \boldsymbol{\nabla}\left\langle c_{A}\right\rangle^{f}+\left\langle c_{A}\right\rangle^{f} \boldsymbol{\nabla} \varepsilon+\frac{1}{V} \int_{\mathrm{A}_{s f}} \mathbf{n} c_{A} \mathrm{~d} A\right)\right] \\
& -k_{1} \alpha_{A} a_{v}\left\langle c_{A}\right\rangle_{s f} \\
\varepsilon \frac{\partial\left\langle c_{B}\right\rangle^{f}}{\partial t}= & \boldsymbol{\nabla} \cdot\left[\mathcal{D}_{B}\left(\varepsilon \boldsymbol{\nabla}\left\langle c_{B}\right\rangle^{f}+\left\langle c_{B}\right\rangle^{f} \boldsymbol{\nabla} \varepsilon+\frac{1}{V} \int_{\mathrm{A}_{s f}} \mathbf{n} c_{B} \mathrm{~d} A\right)\right] \\
& +k_{1} \alpha_{A} a_{v}\left\langle c_{A}\right\rangle_{s f}-k_{2} \alpha_{B} a_{v}\left\langle c_{B}\right\rangle_{s f}
\end{aligned}
$$

where $a_{v}$ is the specific area given by $a_{v}=\frac{A_{s f}}{V}$ and $\left\langle c_{i}\right\rangle_{s f}$ the area average of $c_{i}$ defined by

$$
\left\langle c_{i}\right\rangle_{s f}=\frac{1}{A_{s f}} \int_{\mathrm{A}_{s f}} c_{i} \mathrm{~d} A, \quad i=A, B
$$


The averaged equations (A.3) contain both intrinsic average and pointwise concentrations. To remove the latter, the spatial decomposition

$$
c_{i}=\left\langle c_{i}\right\rangle^{f}+\tilde{c}_{i}, \quad i=A, B
$$

is introduced [46], where $\tilde{c}_{i}$ is the deviation of concentration which fluctuates at a typical length-scale $\ell_{p}$ while $\left\langle c_{i}\right\rangle^{f}$ experiences significant variations at the scale $L$. A consequence of the fact that $r_{0} \ll L$ is that $\left\langle\tilde{c}_{i}\right\rangle^{f} \simeq 0$. Inserting the above decomposition into Eqs. (A.3) leads to

$$
\begin{aligned}
\varepsilon \frac{\partial\left\langle c_{A}\right\rangle^{f}}{\partial t}= & \boldsymbol{\nabla} \cdot\left[\mathcal { D } _ { A } \left(\varepsilon \boldsymbol{\nabla}\left\langle c_{A}\right\rangle^{f}+\left\langle c_{A}\right\rangle^{f} \boldsymbol{\nabla} \varepsilon+\frac{1}{V} \int_{\mathrm{A}_{s f}} \mathbf{n}\left\langle c_{A}\right\rangle^{f} \mathrm{~d} A\right.\right. \\
& \left.\left.+\frac{1}{V} \int_{\mathrm{A}_{s f}} \mathbf{n} \tilde{c}_{A} \mathrm{~d} A\right)\right]-k_{1} \alpha_{A} a_{v}\left(\left\langle\tilde{c}_{A}\right\rangle_{s f}+\left\langle\left\langle c_{A}\right\rangle^{f}\right\rangle_{s f}\right) \\
\varepsilon \frac{\partial\left\langle c_{B}\right\rangle^{f}}{\partial t}= & \boldsymbol{\nabla} \cdot\left[\mathcal { D } _ { B } \left(\varepsilon \boldsymbol{\nabla}\left\langle c_{B}\right\rangle^{f}+\left\langle c_{B}\right\rangle^{f} \boldsymbol{\nabla} \varepsilon+\frac{1}{V} \int_{\mathrm{A}_{s f}} \mathbf{n}\left\langle c_{B}\right\rangle^{f} \mathrm{~d} A\right.\right. \\
& \left.\left.+\frac{1}{V} \int_{\mathrm{A}_{s f}} \mathbf{n} \tilde{c}_{B} \mathrm{~d} A\right)\right]+k_{1} \alpha_{A} a_{v}\left(\left\langle\tilde{c}_{A}\right\rangle_{s f}+\left\langle\left\langle c_{A}\right\rangle^{f}\right\rangle_{s f}\right) \\
& -k_{2} \alpha_{B} a_{v}\left(\left\langle\tilde{c}_{B}\right\rangle_{s f}+\left\langle\left\langle c_{B}\right\rangle^{f}\right\rangle_{s f}\right)
\end{aligned}
$$

The intrinsic average, $\left\langle c_{i}\right\rangle^{f}, i=A, B$, in the area integral terms may be developed around the centroid of the averaging domain using a Taylor expansion. The $0^{\text {th }}$-order terms of these expansions cancel with the terms $\left\langle c_{i}\right\rangle^{f} \boldsymbol{\nabla} \varepsilon$ while order of magnitude estimates can be employed to show that the higher order terms are much smaller than $\varepsilon \boldsymbol{\nabla}\left\langle c_{i}\right\rangle^{f}$ (see Chapter 1 in [34] for the details). In addition, it can be shown that, whenever the pore-scale kinetic number $K i=\ell_{p} \max \left(\frac{k_{1} \alpha_{A}}{\mathcal{D}_{A}}, \frac{k_{2} \alpha_{B}}{\mathcal{D}_{B}}\right)$ satisfies the constraint $K i \ll 1$, then $\tilde{c}_{i} \ll\left\langle c_{i}\right\rangle^{f}, i=A, B$ at $\mathrm{A}_{s f}$ [34. Moreover, by estimating the orders of magnitude of $\left\langle\left\langle c_{i}\right\rangle^{f}\right\rangle_{s f}$, it can be proven that $\left\langle\left\langle c_{i}\right\rangle^{f}\right\rangle_{s f} \approx\left\langle c_{i}\right\rangle^{f}, i=A, B$ [34. As a consequence Eqs. (A.6) are reduced to the following forms

$$
\begin{aligned}
\varepsilon \frac{\partial\left\langle c_{A}\right\rangle^{f}}{\partial t}= & \boldsymbol{\nabla} \cdot\left[\mathcal{D}_{A}\left(\varepsilon \boldsymbol{\nabla}\left\langle c_{A}\right\rangle^{f}+\frac{1}{V} \int_{\mathbf{A}_{s f}} \mathbf{n} \tilde{c}_{A} \mathrm{~d} A\right)\right]-k_{1} \alpha_{A} a_{v}\left\langle c_{A}\right\rangle^{f} \\
\varepsilon \frac{\partial\left\langle c_{B}\right\rangle^{f}}{\partial t}= & \boldsymbol{\nabla} \cdot\left[\mathcal{D}_{B}\left(\varepsilon \boldsymbol{\nabla}\left\langle c_{B}\right\rangle^{f}+\frac{1}{V} \int_{\mathrm{A}_{s f}} \mathbf{n} \tilde{c}_{B} \mathrm{~d} A\right)\right]+k_{1} \alpha_{A} a_{v}\left\langle c_{A}\right\rangle^{f} \\
& -k_{2} \alpha_{B} a_{v}\left\langle c_{B}\right\rangle^{f}
\end{aligned}
$$


At this stage, the model remains unclosed since $\tilde{c}_{i}$ is still present in the averaged mass balance equations. The aim is now to develop a closure, i.e. relationships between $\tilde{c}_{i}$ and $\left\langle c_{i}\right\rangle^{f}$. This can be achieved by subtracting Eqs. A.7) from the initial pore-scale equations (5a) together with the interfacial boundary conditions in order to derive an initial boundary value problem for $\tilde{c}_{i}$. When order of magnitude estimates are employed and under the constraint that the process is considered at a time scale such that

$$
\frac{t}{\ell_{p}^{2}} \min \left(\mathcal{D}_{i}\right) \gg 1 \quad i=A, B
$$

it is not hard to deduce that the closure problems on $\tilde{c}_{A}$ and $\tilde{c}_{B}$ can be simplified to

$$
\begin{aligned}
& \nabla^{2} \tilde{c}_{A}=-\underbrace{\frac{\varepsilon^{-1} k_{1} \alpha_{A} a_{v}}{\mathcal{D}_{A}}\left\langle c_{A}\right\rangle^{f}}_{\text {reactive volume source }} \text { in } \mathrm{V}_{f} \\
& -\mathbf{n} \cdot \mathcal{D}_{A} \boldsymbol{\nabla} \tilde{c}_{A}=\underbrace{\mathbf{n} \cdot \mathcal{D}_{A} \boldsymbol{\nabla}\left\langle c_{A}\right\rangle^{f}}_{\begin{array}{c}
\text { diffusive surface } \\
\text { source }
\end{array}}+\underbrace{k_{1} \alpha_{A}\left\langle c_{A}\right\rangle^{f}}_{\begin{array}{c}
\text { reactive surface } \\
\text { source }
\end{array}} \text { at } \mathrm{A}_{s f} \\
& \left\langle\tilde{c}_{A}\right\rangle^{f}=0 \\
& \tilde{c}_{A}(\mathbf{r})=\tilde{c}_{A}\left(\mathbf{r}+\ell_{i} \mathbf{e}_{i}\right) \quad i=1,2,3 \\
& \nabla^{2} \tilde{c}_{B}=\underbrace{\frac{\varepsilon^{-1} k_{1} \alpha_{A} a_{v}}{\mathcal{D}_{B}}\left\langle c_{A}\right\rangle^{f}}_{\begin{array}{c}
\text { reactive volume source } \\
\text { from species } A
\end{array}}-\underbrace{\frac{\varepsilon^{-1} k_{2} \alpha_{B} a_{v}}{\mathcal{D}_{B}}\left\langle c_{B}\right\rangle^{f}}_{\begin{array}{c}
\text { reactive volume source } \\
\text { from species B }
\end{array}} \text { in } \mathrm{V}_{f} \\
& -\mathbf{n} \cdot \mathcal{D}_{B} \boldsymbol{\nabla} \tilde{c}_{B}=\underbrace{\mathbf{n} \cdot \mathcal{D}_{B} \boldsymbol{\nabla}\left\langle c_{B}\right\rangle^{f}}_{\begin{array}{c}
\text { diffusive surface } \\
\text { source }
\end{array}}-\underbrace{k_{1} \alpha_{A}\left\langle c_{A}\right\rangle^{f}}_{\begin{array}{c}
\text { reative surfae source } \\
\text { from species A }
\end{array}} \\
& +\underbrace{k_{2} \alpha_{B}\left\langle c_{B}\right\rangle^{f}}_{\begin{array}{c}
\text { reactive surface source } \\
\text { from species } \mathrm{B}
\end{array}} \text { at } \mathrm{A}_{s f} \\
& \left\langle\tilde{c}_{B}\right\rangle^{f}=0 \\
& \tilde{c}_{B}(\mathbf{r})=\tilde{c}_{B}\left(\mathbf{r}+\ell_{i} \mathbf{e}_{i}\right) \quad i=1,2,3
\end{aligned}
$$

Since the objective is not to solve these closure problems over the whole structure of size $L$, and with the idea that the boundary condition at $\mathrm{A}_{f e}$ 
371

plays a significant role at a distance of the order of $\ell_{p}$, the solution can be sought on a Representative Elementary Volume of the medium that is considered as periodic with a period $\ell_{i} \mathbf{e}_{i}$ in the $i^{\text {th }}$-direction $(i=1,2,3)$ so that the external boundary condition is replaced by periodic conditions expressed in Eqs. (A.9d) and (A.10d). It should be noted that periodicity is introduced as convenient boundary conditions at the closure level but does not restrict the applicability of the macroscopic model to periodic structures.

Because the two above problems on $\tilde{c}_{A}$ and $\tilde{c}_{B}$ are linear, their solutions can be sought in terms of linear combinations of the sources identified in Eqs. (A.9) and A.10). This allows writing the formal solutions as

$$
\begin{aligned}
& \tilde{c}_{A}=\mathbf{b} \cdot \nabla\left\langle c_{A}\right\rangle^{f}+s_{1}\left\langle c_{A}\right\rangle^{f} \\
& \tilde{c}_{B}=\mathbf{b} \cdot \nabla\left\langle c_{B}\right\rangle^{f}+s_{2}\left\langle c_{A}\right\rangle^{f}+s_{3}\left\langle c_{B}\right\rangle^{f}
\end{aligned}
$$

where $\mathbf{b}, s_{1}, s_{2}$ and $s_{3}$ are the closure variables. When these formal solutions are introduced in the closure problems in Eqs. A.9 and (A.10), and when the contributions from each source are separated, the closure variables are found to obey the four following closure problems

Problem I

$$
\begin{aligned}
\nabla^{2} \mathbf{b} & =0 \quad \text { in } \mathrm{V}_{f} \\
\mathbf{n} \cdot \boldsymbol{\nabla} \mathbf{b} & =-\mathbf{n} \quad \text { at } \mathrm{A}_{s f} \\
\langle\mathbf{b}\rangle^{f} & =0 \\
\mathbf{b}(\mathbf{r}) & =\mathbf{b}\left(\mathbf{r}+\ell_{i} \mathbf{e}_{i}\right)
\end{aligned}
$$

\section{Problem II}

$$
\begin{aligned}
& \nabla^{2} s_{1}=-\frac{\varepsilon^{-1} k_{1} \alpha_{A} a_{v}}{\mathcal{D}_{A}} \quad \text { in } \mathrm{V}_{f} \\
& \mathbf{n} \cdot \nabla s_{1}=-\frac{k_{1} \alpha_{A}}{\mathcal{D}_{A}} \quad \text { at } \mathrm{A}_{s f} \\
& \left\langle s_{1}\right\rangle^{f}=0 \\
& s_{1}(\mathbf{r})=s_{1}\left(\mathbf{r}+\ell_{i} \mathbf{e}_{i}\right)
\end{aligned}
$$


Problem III

$$
\begin{aligned}
\nabla^{2} s_{2} & =\frac{\varepsilon^{-1} k_{1} \alpha_{A} a_{v}}{\mathcal{D}_{B}} \quad \text { in } \mathrm{V}_{f} \\
\mathbf{n} \cdot \boldsymbol{\nabla} s_{2} & =\frac{k_{1} \alpha_{A}}{\mathcal{D}_{B}} \quad \text { at } \mathrm{A}_{s f} \\
\left\langle s_{2}\right\rangle^{f} & =0 \\
s_{2}(\mathbf{r}) & =s_{2}\left(\mathbf{r}+\ell_{i} \mathbf{e}_{i}\right)
\end{aligned}
$$

Problem IV

$$
\begin{aligned}
& \nabla^{2} s_{3}=-\frac{\varepsilon^{-1} k_{2} \alpha_{B} a_{v}}{\mathcal{D}_{B}} \quad \text { in } \mathrm{V}_{f} \\
& \mathbf{n} \cdot \boldsymbol{\nabla} s_{3}=-\frac{k_{2} \alpha_{B}}{\mathcal{D}_{B}} \quad \text { at } \mathrm{A}_{s f} \\
& \left\langle s_{3}\right\rangle^{f}=0 \\
& s_{3}(\mathbf{r})=s_{3}\left(\mathbf{r}+\ell_{i} \mathbf{e}_{i}\right)
\end{aligned}
$$

Inserting the representations of $\tilde{c}_{A}$ and $\tilde{c}_{B}$ into the average equation A.7) finally yields the closed macroscopic mass balance equations

$$
\begin{aligned}
\varepsilon \frac{\partial\left\langle c_{A}\right\rangle^{f}}{\partial t}= & \boldsymbol{\nabla} \cdot\left(\varepsilon \mathcal{D}_{A} \mathbf{D}_{e f f}^{*} \cdot \nabla\left\langle c_{A}\right\rangle^{f}\right)+\boldsymbol{\nabla} \cdot\left(\mathbf{u}_{1}\left\langle c_{A}\right\rangle^{f}\right) \\
& -k_{1} \alpha_{A} a_{v}\left\langle c_{A}\right\rangle^{f} \\
\varepsilon \frac{\partial\left\langle c_{B}\right\rangle^{f}}{\partial t}= & \boldsymbol{\nabla} \cdot\left(\varepsilon \mathcal{D}_{B} \mathbf{D}_{\text {eff }}^{*} \cdot \boldsymbol{\nabla}\left\langle c_{A}\right\rangle^{f}\right)+\boldsymbol{\nabla} \cdot\left(\mathbf{u}_{2}\left\langle c_{A}\right\rangle^{f}\right) \\
& +\boldsymbol{\nabla} \cdot\left(\mathbf{u}_{3}\left\langle c_{B}\right\rangle^{f}\right)+k_{1} \alpha_{A} a_{v}\left\langle c_{A}\right\rangle^{f}-k_{2} \alpha_{B} a_{v}\left\langle c_{B}\right\rangle^{f}
\end{aligned}
$$

with the effective parameters $\mathbf{D}_{e f f}^{*}, \mathbf{u}_{1}, \mathbf{u}_{2}$ and $\mathbf{u}_{3}$ given by

$$
\begin{gathered}
\mathbf{D}_{e f f}^{*}=\mathbf{I}+\frac{1}{V_{f}} \int_{\mathrm{A}_{s f}} \mathbf{n b} d A \\
\mathbf{u}_{1}=\frac{\mathcal{D}_{A}}{V}\left(\int_{\mathbf{A}_{s f}} \mathbf{n} s_{1} d A\right) ; \quad \mathbf{u}_{2}=\frac{\mathcal{D}_{B}}{V}\left(\int_{\mathrm{A}_{s f}} \mathbf{n} s_{2} d A\right) ; \\
\mathbf{u}_{3}=\frac{\mathcal{D}_{B}}{V}\left(\int_{\mathrm{A}_{s f}} \mathbf{n} s_{3} d A\right)
\end{gathered}
$$


From Eqs. A.13b, A.14b and A.15b), the order of magnitude estimates of the closure variables $s_{1}, s_{2}$ and $s_{3}$ at $\mathrm{A}_{s f}$ can be extracted to give

$$
s_{1}=\mathrm{O}\left(\frac{l_{p} k_{1} \alpha_{A}}{\mathcal{D}_{A}}\right) ; \quad s_{2}=\mathrm{O}\left(\frac{l_{p} k_{1} \alpha_{A}}{\mathcal{D}_{B}}\right) ; \quad s_{3}=\mathrm{O}\left(\frac{l_{p} k_{2} \alpha_{B}}{\mathcal{D}_{B}}\right)
$$

Once introduced in Eqs. A.17b), this leads to the following order of magnitude estimates

$$
\begin{aligned}
\boldsymbol{\nabla} \cdot\left(\mathbf{u}_{\mathbf{1}}\left\langle c_{A}\right\rangle^{f}\right)= & \mathbf{O}\left(\frac{k_{1} \alpha_{A}}{L}\left\langle c_{A}\right\rangle^{f}\right) ; \quad \boldsymbol{\nabla} \cdot\left(\mathbf{u}_{\mathbf{2}}\left\langle c_{A}\right\rangle^{f}\right)=\mathbf{O}\left(\frac{k_{1} \alpha_{A}}{L}\left\langle c_{A}\right\rangle^{f}\right) ; \\
& \boldsymbol{\nabla} \cdot\left(\mathbf{u}_{\mathbf{3}}\left\langle c_{B}\right\rangle^{f}\right)=\mathbf{O}\left(\frac{k_{2} \alpha_{B}}{L}\left\langle c_{B}\right\rangle^{f}\right)
\end{aligned}
$$

However, the order of magnitude estimates for the macroscopic reactive terms are

$$
k_{1} \alpha_{A} a_{v}\left\langle c_{A}\right\rangle^{f}=\mathbf{O}\left(\frac{k_{1} \alpha_{A}}{\ell_{p}}\left\langle c_{A}\right\rangle^{f}\right) ; \quad k_{2} \alpha_{B} a_{v}\left\langle c_{B}\right\rangle^{f}=\mathbf{O}\left(\frac{k_{2} \alpha_{B}}{\ell_{p}}\left\langle c_{B}\right\rangle^{f}\right)
$$

In the latter, $a_{v}$ was estimated to be $a_{v}=\mathbf{O}\left(\ell_{p}^{-1}\right)$. From Eqs. A.19) and A.20, and due to the length-scale hierarchy $\ell_{p} \ll L$, it follows that the macroscopic diffusion/reaction equations (A.16) can be written in their final simplified forms as

$$
\begin{aligned}
& \varepsilon \frac{\partial\left\langle c_{A}\right\rangle^{f}}{\partial t}=\nabla \cdot\left(\varepsilon \mathcal{D}_{A} \mathbf{D}_{e f f}^{*} \cdot \nabla\left\langle c_{A}\right\rangle^{f}\right)-k_{1} \alpha_{A} a_{v}\left\langle c_{A}\right\rangle^{f} \\
& \varepsilon \frac{\partial\left\langle c_{B}\right\rangle^{f}}{\partial t}=\boldsymbol{\nabla} \cdot\left(\varepsilon \mathcal{D}_{B} \mathbf{D}_{e f f}^{*} \cdot \nabla\left\langle c_{A}\right\rangle^{f}\right)+k_{1} \alpha_{A} a_{v}\left\langle c_{A}\right\rangle^{f} \\
& -k_{2} \alpha_{B} a_{v}\left\langle c_{B}\right\rangle^{f}
\end{aligned}
$$

It is worth noting that, after the simplifications resulting from the order of magnitude estimates, the macroscopic mass balance equations A.21 for the two species only require the solution of the closure problem I in Eqs. A.12 yielding the effective diffusivity tensor $\mathbf{D}_{\text {eff }}^{*}$ given by Eq. A.17a.

[1] D. Leech, P. Kavanagh, W. Schuhmann, Enzymatic fuel cells: Recent progress, Electrochim. Acta. 84 (2012) 223-234. 
[2] S. Cosnier, A. J. Gross, A. L. Goff, M. Holzinger, Recent advances on enzymatic glucose/oxygen and hydrogen/oxygen biofuel cells: Achievements and limitations, J. Pow. Sc. 325 (2016) 252-263.

[3] M. Gamella, A. Koushanpour, E. Katz, Biofuel cells - activation of micro- and macro-electronic devices, Bioelectrochemistry 119 (2018) 3342 .

[4] S. Reculusa, M. Heim, F. Gao, N. Mano, S. Ravaine, A. Kuhn, Design of catalytically active cylindrical and macroporous gold microelectrodes, Adv. Funct. Mater. 21 (4) (2011) 691-698. doi:10.1002/adfm. 201001761 .

[5] A. Karajić, S. Reculusa, M. Heim, P. Garrigue, S. Ravaine, N. Mano, A. Kuhn, Bottom-up generation of miniaturized coaxial double electrodes with tunable porosity, Adv. Mater. Interfaces 2 (12) (2015) 1500192-1500196. doi:10.1002/admi.201500192.

[6] A. Karajić, S. Reculusa, S. Ravaine, N. Mano, A. Kuhn, Miniaturized electrochemical device from assembled cylindrical macroporous gold electrodes, ChemElectroChem 03 (2016) 2031-2035.

[7] A. Walcarius, A. Kuhn, Ordered porous thin films in electrochemical analysis, Trends Anal. Chem. 27 (2008) 593-603.

[8] P. N. Bartlett, T. F. Esterle, The oxidation of hydrogen peroxide on nanostructured rhodium microelectrodes, J. Electrochemistry 18 (2012) 457-471. doi:1006-3471(2012)05-0457-15.

[9] T. Q. N. Do, M. Varničić, R. Hanke-Rauschenbach, T. Vidaković-Koch, K. Sundmacher, Mathematical modeling of a porous enzymatic electrode with direct electron transfer mechanism, Electrochim. Acta 137 (2014) 616-626. doi:10.1016/j.electacta.2014.06.031

[10] T. Vidakovic-Koch, K. Sundmacher, Porous electrodes in bioelectrochemistry, in: K. Wandelt (Ed.), Encyclopedia of Interfacial Chemistry, Elsevier, Oxford, 2018, pp. $392-401$. doi :https://doi.org/10.1016/ B978-0-12-409547-2.13522-X. 
[11] J. Galceran, S. L. Taylor, P. N. Bartlett, Modelling the steady-state current at the inlaid disc microelectrode for homogeneous mediated enzyme catalysed reactions, J. Electroanal. Chem. 506 (2001) 65-81.

[12] T. Q. N. Do, M. Varničić, R. Flassig, T. Vidaković-Koch, K. Sundmacher, Dynamic and steady state 1-d model of mediated electron transfer in a porous enzymatic electrode, Bioelectrochemistry 106 (2015) 313 .

[13] T. D. Le, D. Lasseux, X. P. Nguyen, G. L. Vignoles, N. Mano, A. Kuhn, Multi-scale modeling of diffusion and electrochemical reactions in porous micro-electrodes, Chem. Eng. Sci. 173 (2017) 153-167.

[14] T. D. Le, L. Zhang, G. L. Vignoles, N. Mano, A. Kuhn, D. Lasseux, Optimal thickness of a porous micro-electrode operating a single redox reaction, ChemElectroChem 6 (2019) 173-180.

[15] R. D. Levie, Electrochemical response of porous and rough electrodes, Adv. Electrochem. Electrochem. Eng. 6 (1967) 329-397.

[16] O. E. Barcia, E. D’Elia, I. Frateur, O. R. Mattos, N. Pebere, B. Tribollet, Application of the impedance model of de Levie for the characterization of porous electrodes, Electrochim. Acta 47 (2002) 2109-2116.

[17] E. O. Barnes, X. Chen, P. Li, R. G. Compton, Voltammetry at porous electrodes: A theoretical study, J. Electroanal. Chem. 720-721 (2014) 92-100.

[18] Z. Ban, E. Katelhon, R. G. Compton, Voltammetry of porous layers: Staircase vs analog voltammetry, J. Electroanal. Chem. 776 (2016) 2533 .

[19] H. T. H. Chan, E. Katelhon, R. G. Compton, Voltammetry using multiple cycles: Porous electrodes, J. Electroanal. Chem. 799 (2017) 126-133.

[20] M. Ender, An extended homogenized porous electrode model for lithiumion cell electrodes, J. Power Sources 282 (2015) 572-580.

[21] T. R. Ferguson, M. Z. Bazant, Nonequilibrium thermodynamics of porous electrodes, J. Electrochem. Soc. 159 (2012) 1967-1985. 
[22] R. T. Bonnecaze, N. Mano, B. Nam, A. Heller, On the behavior of the porous rotating disk electrode, J. Electrochem. Soc. 154 (2007) 44-47.

[23] B. Nam, R. T. Bonnecaze, Analytic models of the infinite porous rotating disk electrode, J. Electrochem. Soc. 154 (2017) 191-197.

[24] P. D. Vidts, R. E. White, Governing equations for transport in porous electrodes, J. Electrochem. Soc. 144 (1997) 1343-1352.

[25] F. Yin, Y. Liu, C. Wang, H. Liu, Assessing the electron transfer and oxygen mass transfer of the oxygen reduction reaction using a new electrode kinetic equation, Phys. Chem. Chem. Phys. 20 (2018) 16159-16166.

[26] G. Gotti, K. Fajerwerg, D. Evrard, P. Gros, Kinetics of dioxygen reduction on gold and glassy carbon electrodes in neutral media, Int. J. Electrochem. Sci. 8 (2013) 12643-12657.

[27] J. S. Jirkovsky, M. Halasa, D. J. Schiffrin, Kinetics of electrocatalytic reduction of oxygen and hydrogen peroxide on dispersed gold nanoparticles, Phys. Chem. Chem. Phys. 12 (2010) 8042-8052.

[28] P. Vassilev, M. T. M. Koper, Electrochemical reduction of oxygen on gold surfaces: a density functional theory study of intermediates and reaction paths, J. Phys. Chem. C. 111 (2007) 2607-2613.

[29] R. Zeis, T. Lei, K. Sieradzki, J. Snyder, J. Erlebacher, Catalytic reduction of oxygen and hydrogen peroxide by nanoporous gold, J. Catal. 253 (2008) 132-138.

[30] M. S. El-Deab, T. Ohsaka, An extraordinary electrocatalytic reduction of oxygen on gold nanoparticles-electrodeposited gold electrodes, Electrochemistry Communications 4 (2002) 288-292.

[31] J. Kim, A. A. Gewirth, Mechanism of oxygen electroreduction on gold surfaces in basic media, Journal of Physical Chemistry B 110 (2006) 2565-2571.

[32] A. Fick, On liquid diffusion, J. Membr. Sci. 100 (1995) 33-38.

[33] J. A. V. Butler, The mechanism of overvoltage and its relation to the combination of hydrogen atoms at metal electrodes, Trans. Faraday Soc. 28 (1932) 379-382. 
[34] S. Whitaker, The method of volume averaging, Kluwer Academic Publishers, Dordrecht, The Netherlands, 1999.

[35] S. Whitaker, Derivation and application of the Stefan-Maxwell equations, Rev. Mex. Ing. Chim. 18 (3) (2009) 213-244.

[36] S. Whitaker, F. Valdés-Parada, Private communication (2019).

[37] S. A. M. Biezen, F. M. Everaerts, L. J. J. Janssen, R. A. Tacken, Diffusion coefficients of oxygen, hydrogen peroxide and glucose in a hydrogel, Anal. Chim. Acta 273 (1993) 553-560.

[38] T. Shinagawa, A. T. Garcia-Esparza, K.Takanabe, Insight on tafel slopes from a microkinetic analysis of aqueous electrocatalysis for energy conversion, Sci. Rep. 5:13801 (2015) 1-21. doi:DOI:10.1038/srep13801.

[39] J. Zhang, PEM Fuel Cell Electrocatalysts and Catalyst Layers, chapter Electrocatalytic Oxygen Reduction Reaction, Springer London, 2008.

[40] S. Reculusa, S. Ravaine, Synthesis of colloidal crystals of controllable thickness through the Langmuir-Blodgett technique, Chem. Mater. 15 (2003) 598-605. doi:10.1021/cm021242w.

[41] R. Szamocki, S. Reculusa, S. Ravaine, P. N. Bartlett, A. Kuhn, R. Hempelmann, Tailored mesostructuring and biofunctionalization of gold for increased electroactivity, Angewandte Chemie International Edition 45 (8) (2006) 1317-1321. doi:10.1002/anie.200503292.

[42] M. Heim, S. Reculusa, S. Ravaine, A. Kuhn, Engineering of complex macroporous materials through controlled electrodeposition in colloidal superstructures, Adv. Funct. Mater. 22 (3) (2012) 538-545. doi:10. 1002/adfm. 201101918.

[43] P. N. Bartlett, P. R. Birkina, M. A. Ghanema, Electrochemical deposition of macroporous platinum, palladium and cobalt films using polystyrene latex sphere templates, Chem. Commun. 17 (2000) 16711672. doi:10.1039/B004398M.

[44] C. Truesdell, R. Toupin, The classical field theories, Springer-Verlag, New-York, 1960. 
499

[45] F. Howes, S. Whitaker, The spatial averaging theorem revisited, Chem. Eng. Sci. 40 (1985) 1387-1392. doi:10.1016/0009-2509(85)80078-6.

[46] W. G. Gray, A derivation of the equations for multi-phase transport, Chem. Eng. Sci. 30 (1975) 229-233. 